Huang et al., Afr J Tradit Complement Altern Med., (2018) 15 (1): 98-109

https://doi.org/10.21010/ajtcam.v15i1.10

\title{
ELECTRO-ACUPUNCTURE AT JIANSHI (PC5) AND NEIGUAN (PC6) ALTERS HEART RATE VARIABILITY (HRV) IN FRIGHTENED VOLUNTEERS
}

\section{Huanlin Huang" ${ }^{1 \#}$, Zheng Zhong ${ }^{2 \#}$, Xinyi Zhang ${ }^{1}$, Jizhou Wang ${ }^{3}$, Yujie $\mathrm{Li}^{3}$, Ole Bernt Fasmer ${ }^{4}$, Gustav Wik $^{4}$, Yong Huang ${ }^{*}$, Shaohui Huang ${ }^{1}$.}

${ }^{1}$ School of Traditional Chinese Medicine, Southern Medical University, Guangzhou, Guangdong, China;

${ }^{2}$ Department of Traditional Chinese Medicine, Nanfang Hospital, Southern Medical University, Guangzhou,

Guangdong, China; ${ }^{3}$ The First School of Clinical Medicine, Southern Medical University, Guangzhou,

Guangdong, China; ${ }^{4}$ Faculty of Medicine and Dentistry, Department of Clinical Medicine, University of

Bergen, Bergen, Norway. "Huanlin Huang and Zheng Zhong contributed equally to this work.

*Corresponding Authors E-mail: nanfanglihuang@163.com (YH); gustav.wik@kliniskmedisin.uib.no $(\mathrm{GW})$; hshtcm@126.com $(\mathrm{SH})$

\begin{tabular}{|c|}
\hline \multicolumn{1}{|c|}{ Article History } \\
Received: Jan. 18, 2017. \\
Revised Received: Sept. 09, 2017. \\
Accepted: Sept. 13, 2017. \\
Published Online: Dec. 29, 2017. \\
\hline
\end{tabular}

\begin{abstract}
Background: Fear is one of the most widely studied emotions and is closely associated with the autonomic nervous system (ANS). Previous studies have proven that acupuncture directly impacts the ANS, influences the heart rate (HR) and the heart rate variability (HRV) and exerts other effects. The aim of this study was to explore the effect of Jianshi (PC5) and Neiguan (PC6) electro-acupuncture on HRV during fear-invoking auditory stimulation using an Actiheart ECG recorder.
\end{abstract}

Materials and Methods: Two hundred healthy subjects were recruited. Using a random number table, subjects were grouped for exposure to fear-invoking auditory stimulation $(n=40)$ or neutral auditory stimulation $(n=40)$. After determining that our fear-invoking auditory stimulation produced the fear emotion, the other 120 subjects were similarly divided into an electro-acupuncture (EA group) and a control group that received PC5 and PC6 electro-acupuncture or no intervention.

Results: The fear score of the fear-invoking auditory group was significantly higher than that of the neutral auditory group. The EA group showed higher SD, RMSSD, and high frequency (HF) components of HRV than those of the control group.

Conclusion: The primary result suggests that PC5 and PC6 electro-acupuncture affects cardiac autonomic neural regulation, mainly via the parasympathetic system, in subjects exposed to fear-invoking auditory stimulation.

Keywords: electro-acupuncture; Jianshi (PC5); Neiguan (PC6); Heart Rate Variability (HRV); fear emotion; Autonomic Nervous System (ANS) 


\section{Introduction}

Fear may be the most widely studied emotion (Gullone et al., 1996). It was systematically described by Charles Robert Darwin in The expression of the emotions in man and animals (1872). Studies from Brokovec and O'Brien (Borkovec and O'Brien, 1977), Lerner (Momen et al., 2005), and Etzel (Etzel et al., 2006) have reported an increase in heart rate (HR) during fear. HR changes are in response to the activation or suppression of the cardiac autonomic nervous system (ANS), which is synergistically mediated by the cardiac sympathetic and parasympathetic systems (Guo and Zhang, 2007). An increasing HR indicates cardiac sympathetic system activation or parasympathetic system suppression. Studies have suggested that the main effects of acupuncture include HR reduction and heart rate variability (HRV) alteration (Hsu et al., 2007).

HRV specifies the variations that occur within every cardiac cycle, as relayed by adjustments from the ANS to the cardiac sinoatrial node. HRV indexes thus reflect the activity balance of the sympathetic and parasympathetic systems (Asl et al., 2008, de la Cruz Torres et al., 2016). In recent years, multiple studies have explored the effect of acupuncture on HRV in physiological and pathological conditions (de la Cruz Torres et al., 2016, Guo and Zhang, 2007, Asl et al., 2008, Jia et al., 2011, Nakahara et al., 2016, B A Cker et al., 2008, Park et al., 2008). One current conclusion is that acupuncture activates the parasympathetic system, which leads to HR reduction (Wang et al., 2002, Litscher, 2010). In a review aimed to explore the mechanisms of acupuncture, Anderson et al. (2012) has confirmed that despite varying research methods and the limitations of experimental conditions, acupuncture can increase HRV, which in turn indicates that acupuncture may reduce the incidence of cardiovascular disorders by balancing ANS activities.

Neiguan (PC6) is one of the most frequently used clinical acupoints (acupuncture points) and is important for treating cardiovascular disorders in clinical practice (Witt et al., 2012). Recent investigations have also indicated that PC6 and Jianshi (PC5) are related to segments of peripheral ganglions relaying information from other internal organs to the heart. PC5 and PC6 converge at the plies of the spinal cord (especially V, VI, VII) in the central pivot (Tao et al., 1993). It is also possible that nervous fibres of the cardiac partitions and those influenced by the PC6 stem from the same or adjacent cells in sympathetic and parasympathetic ganglions (Liu et al., 2002). Nervous impulses produced by PC5 needling have been suggested to pass through median nerves II and III, to be integrated in the spinal cord, and then to directly or indirectly modulate cardiac autonomic nervous functions (Li et al., 2012). Assuming these models describe a morphological basis of acupuncture's effect on the emotion of fear, we decided to study the effect of PC5 and PC6 electro-acupuncture on HRV during fear-invoking auditory stimulation using an Actiheart ECG recorder (Brage et al., 2005).

\section{Materials and Methods \\ Participants}

Two hundred (100 male and 100 female) students from Southern Medical University were recruited from October 2013 to December 2015, with 80 subjects for the audio evocation effect experiment and 120 for the electro-acupuncture experiment. (Fig. 1) 


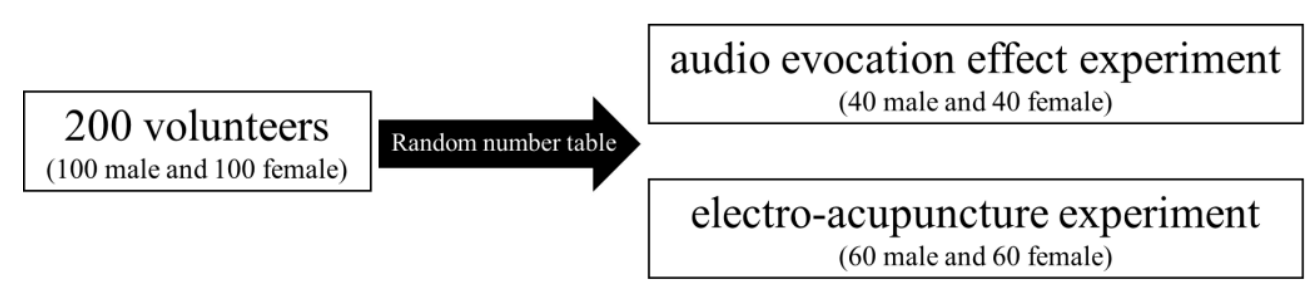

Figure 1: Grouping of the volunteers

\section{Clinical trials registry and ethics review}

This study protocol was approved by the Chinese Registered Clinical Trial Ethics Committee (ChiECRCT-2012008), and written informed consent was obtained from each participant before the study began.

The experiment was performed in accordance with the approved guidelines. The trial was registered in the China Clinical Trials register on $13^{\text {th }}$ March 2012 (ChiCTR-TRC-12002386).

\section{Inclusion and exclusion criteria}

\section{Inclusion criteria}

(1) Undergraduate students aged from 18 to 24 years. (2) Physically healthy, with a body mass index (BMI) index ranging from 18.5 to 22.9 together with a waistline less than $80 \mathrm{~cm}$ for females and $90 \mathrm{~cm}$ for males. (3) Regular diet (no addiction to tea, coffee, alcoholic beverages, or tobacco). (4) Normal ECG and no history of cardiac disease such as viral myocarditis, cardiac arrhythmia, etc. (5) No history (more than 3 months) of any drugs that might affect the HR. (6) No history of any types of pain (including dysmenorrhoea). (7) No fear of electro-acupuncture therapy. (8) Given informed consent for participating in the study.

\section{Exclusion criteria}

(1) History of thrombocytopenia, haemophilia, or coagulation dysfunction. (2) Habit of listening to horror audios or watching horror movies (including those who had such experience within 1 week prior). (3) Smoke, alcohol, or coffee intake within 24 hours prior. (4) Strenuous exercise within 24 hours prior. (5) Incidents of emotional provocations, such as examinations, within 1 week prior. (6) Currently within the menstrual period (females).

\section{Blinding}

Participants were not blinded during the investigational proceedings, but the researchers and statisticians were.

\section{Audio evocation effect experiment}

\section{Emotional evocation}

Only the auditory modality was stimulated to evoke the emotion of fear. All the subjects stayed alone in a quiet dark room, and visual stimulation was blocked by black goggles during the auditory stimulation. 


\section{Audio material}

To evoke the fear emotion, we stimulated our subjects using a scary story and evaluated the effect by the 9-level Likert Scale, which contains 6 emotion descriptions from SAM (Palomba et al., 2000; Bradley and Lang, 1994).

WaveCN (sound-processing software, version 2.0.0.5, Xindong $\mathrm{Su}$, China) was used to edit the audio files. The controlled parameters included a sound frequency of $44100 \mathrm{~Hz}$ in a dual track, the sampling digit was 16 , and the average sound intensity was $22 \mathrm{~dB}$ (Liu et al., 2006).

(1) The neutral audio: Ali Baba and the Forty Thieves from Bedtime Stories (Xue et al., 2009) and a section of The Girl Who Fell from the Sky, making the entire length of the audio 20 minutes.

(2) The fear-invoking audio: Sister's eyes series in Zhangzhen Storytelling and sections of horror sounds from the same series, making the entire length of the audio 20 minutes.

\section{Time and location}

Time: 19:00 to 24:00 from Sunday to Thursday the week following inclusion (avoiding the irregular daily routine caused by a weekend falling on the next day).

Location: A $20 \mathrm{~m}^{2}$ laboratory of acupuncture and moxibustion, located at the Traditional Chinese Medical School in the Southern Medical University. The laboratory was quiet and darkened, with an ambient temperature of $23-27^{\circ} \mathrm{C}$.

\section{Procedure of the audio evocation effect experiment}

The 80 subjects (40 male and 40 female) were divided into a neutral and a fear-invoking group according to a random number table. After the subjects came to the laboratory, they had a 5-minute quiet rest before supplying personal information and signing the informed consent form. Subjects were then asked to lie down on the bed and to put on black goggles (Annan Carbon Technology MRX003-C011; Zhejiang, China) and earphones (SHE2550; Philips Electronic Singapore Pte, Ltd., Toa Payoh, Singapore). The lights were turned off, and no researcher was in the room, which meant the subjects were listening to the fear-invoking/neutral audio alone for 20 minutes. After the stimulation, the subjects were asked to complete the 9-level Likert Scale. All the subjects left after a 20-minute observation to ensure no discomfort occurred (Fig. 2).

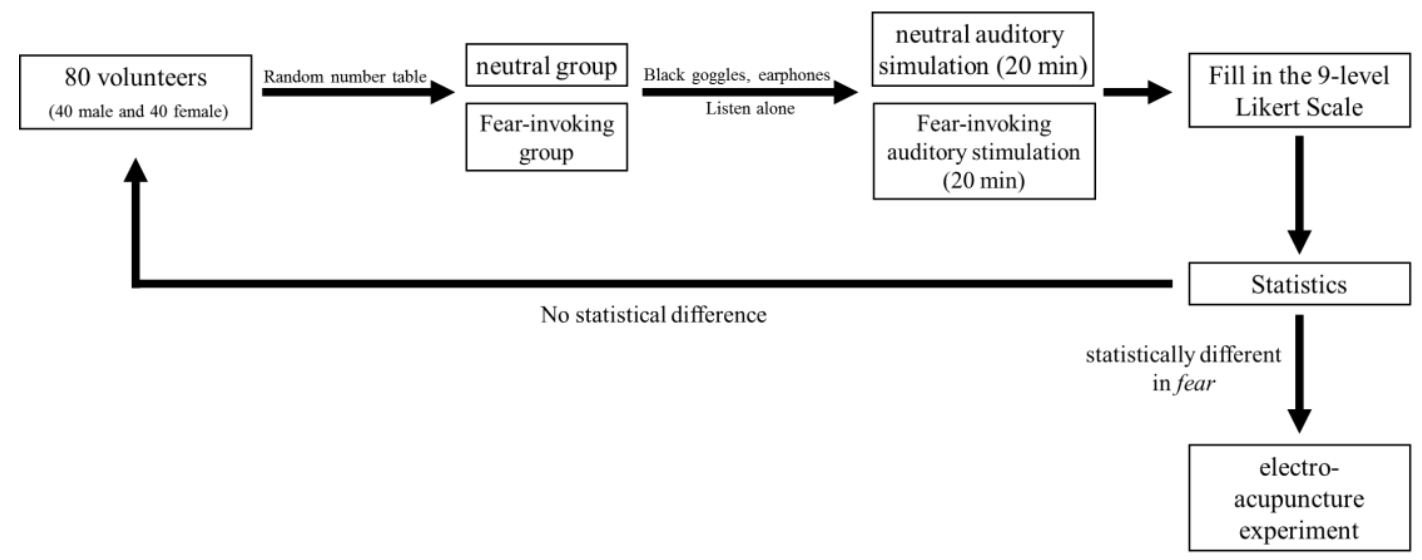

Figure 2: Flow chart of the audio evocation effect experiment 
The Mann-Whitney U Test was used to analyse the data from the 9-level Likert Scale between the two groups in the audio evocation experiment.

\section{Results of the audio evocation effect experiment}

In the neutral group, 3 ECG recordings were interrupted, 2 subjects fell asleep, and 2 subjects changed their positions so that recordings became unclear. In the fear-invoking group, 2 ECG recordings were interrupted, 2 subjects were too frightened to continue the experiment, and 4 subjects changed their positions so that recordings became unclear. These subjects were not included in the statistical analysis. The final number of subjects included was thus 65 (33 from the neutral group and 32 from the fear-invoking group).

The difference in fear level between the two groups showed that the fear-invoking auditory stimulation produced the fear emotion. However, both the neutral and fear-invoking audios induced a certain extent of interest among the subjects, which might be related to interest in the stories. Regarding the interest-related items, there were no differences between the two groups. All the above findings indicated that the fear-invoking auditory stimulation produced the fear emotion (Table 1).

Table 1: Comparison of emotion grades after neutral or fear-invoking auditory stimulation

\begin{tabular}{|c|c|c|c|c|}
\hline Items & $\begin{array}{l}\text { Neutral audio } \\
\text { group }(\mathbf{N}=\mathbf{3 3})\end{array}$ & \begin{tabular}{l}
\multicolumn{2}{l}{ Fear-invoking } \\
audio group \\
$(\mathrm{N}=32)$
\end{tabular} & T value & P value \\
\hline Happiness & $1.91 \pm 1.355$ & $1.75 \pm 1.760$ & -0.747 & 0.455 \\
\hline Sadness & $0.30 \pm 0.637$ & $0.41 \pm 0.560$ & -1.179 & 0.238 \\
\hline Disgust & $0.82 \pm 1.424$ & $0.97 \pm 0.993$ & -1.526 & 0.127 \\
\hline Interest & $3.48 \pm 1.856$ & $3.06 \pm 2.109$ & -1.023 & 0.306 \\
\hline Anger & $0.58 \pm 1.001$ & $0.94 \pm 1.014$ & -1.725 & 0.085 \\
\hline Fear & $1.00 \pm 1.803$ & $4.41 \pm 2.312$ & -5.557 & $0.000 \%$ \\
\hline
\end{tabular}

* $\mathrm{P}<0.05$ comparison between the neutral and fear-invoking groups.

\section{Electro-acupuncture experiment}

The other 120 subjects (60 male and 60 female) here were divided into a control group and an electro-acupuncture group according to a random number table. Each subject participated only once in this experiment and should not have taken part in the experiment above.

\section{Time and location}

Time and location conditions were the same as the audio evocation effect experiment above. 


\section{Procedure of the electro-acupuncture experiment}

The subjects had a 5-minute quiet rest in the laboratory before they completed the personal information forms and then signed the informed consent forms. One of the researchers then recorded their HR and asked the subjects to lie down on the bed in the supine position and to rest quietly for another 5 minutes. The skin of each subject was then cleaned at the level of the xiphoid process; subsequently, electrodes (Ambu blue sensor VL-00-S, Ambu A/S, Ballerup, Malaysia) were attached and connected to the Actiheart recorder (Cambridge Neurotechnology, Ltd.). The HRV was recorded before, during and after the audio, as well as before, during, and after the electro-acupuncture. Before the HRV recording, the PC5 and PC6 were located and marked in each subject's hands in the electro-acupuncture group, in accordance with the WHO Standard Acupuncture Point Location in the Western Pacific Region (World Health Organization, 2008). The skin was then sterilized with $75 \%$ alcohol, and tube needles $(\Phi 0.30 \times 25 \mathrm{~mm}$, Tianxie, Suzhou, China) were inserted to a depth of $15 \pm 2 \mathrm{~mm}$. After the needles were inserted, the tube was carefully removed, the twirling method was adapted to generate a needle sensation.

PC5 and PC6 on the same side were then connected to the Han Electric acupuncture apparatus (LH202H, Huawei Industrial Development Limited Company, Beijing, China), and the stimulus frequency was set at 2/100 $\mathrm{Hz}$ with disperse-dense wave; the current intensity depended on the subject's tolerance or was increased until the skin moved slightly. By contrast, subjects in the control group lay down without any of these interventions. After the stimulation, all subjects remained in the supine position for 20 minutes. All the subjects left after a 20 -minute observation to ensure that no discomfort occurred (Fig. 3).

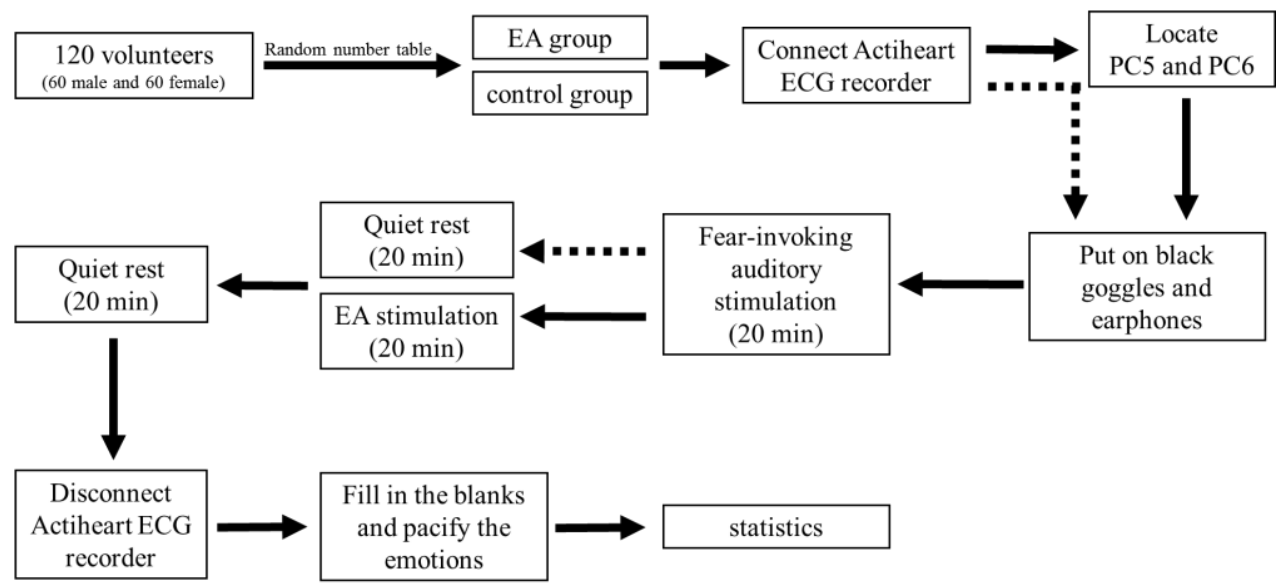

Figure 3: Flow chart of the electro-acupuncture experiment

\section{Observational index}

(1) Heart rate (BPM/HR). (2) Inter-beat interval (IBI). (3) The standard deviation of IBI (SD). (4) Root mean square successive difference of N-to-N intervals (RMSSD). (5) Very low frequency (VLF) (0.0033-0.04 Hz). (6) Low frequency (LF) (0.04-0.15 Hz). (7) High frequency (HF) (0.15-0.40 Hz). (8) Ratio of LF and HF (LF/HF). (9) Triangular index.

Frequency-domain indexes VLF, LF, HF and the LF/HF ratio were obtained using Fourier analysis (Park et al., 2008). 


\section{Statistics}

\section{Data export and processing}

The data were exported to a computer through a short range analysis on Actiheart ECG recorder software. The sampling period of the indexes was set at 5 minutes. The selective time span (Fig. 4) for analysis was 5 minutes before the fear-invoking audio (before audio), the last 5 minutes of the 20-minute audio (after audio), the last 5 minutes of the 20-minute electro-acupuncture (during electro-acupuncture) and the last 5 minutes of the 20-minute quiet rest (after electro-acupuncture).

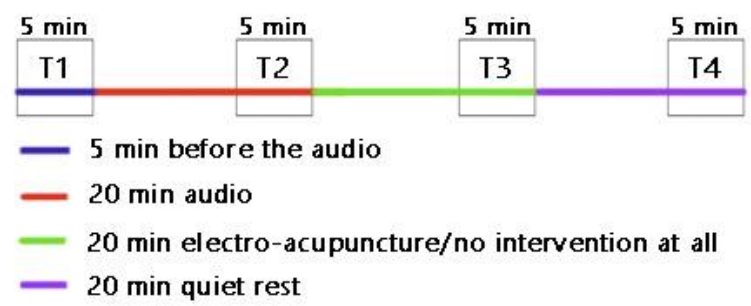

Figure 4: Flow chart of the electro-acupuncture experiment

Data from the four periods (before the audio, after the audio, during electro-acupuncture and after electro-acupuncture) and data of the emotions scale were sorted and processed via MS Excel 2013 (Microsoft Corporation, Redmond, Washington, USA).

\section{Statistical analysis}

A descriptive analysis of the data was performed using the mean $\pm \mathrm{SD}(\mathrm{x} \pm \mathrm{s})$. The frequency-domain indexes VLF, LF and HF were subjected to logarithmic transformation (ln). All analyses were performed using SPSS 20.0 (IBM Corporation, Armonk, New York, USA) by a statistician blinded to the experimental allocation. The Kruskal-Wallis H test was used for data of all emotion items from the 9-level Likert Scale in the electro-acupuncture experiment. Indexes of HRV between the electro-acupuncture group and the control group were analysed using the Bonferroni-corrected Mann-Whitney test.

\section{Results}

\section{Baseline information of the electro-acupuncture experiment}

During the electro-acupuncture experiment, 2 ECGs of the control group were interrupted, 2 subjects fell asleep, 3 subjects changed their positions so that the recordings became unclear, and 2 subjects were too frightened to continue the experiment. In the electro-acupuncture group, 1 ECG recording was interrupted, 1 subject changed his position so that the recordings became indistinct, 1 subject was too frightened to continue, and 3 subjects could not tolerate the minimum quantity of stimulus required for the electro-acupuncture. These subjects were not included in the statistical analysis. The final number of subjects analysed was thus 105 (52 in the control group and 53 in the electro-acupuncture group).

The baseline information of the subjects is listed in Table 2. There were no differences between the two groups in any indexes, including gender, age, height, weight, BMI, HR, and fear grade scores, before the experiment. 
Table 2: Baseline characteristics of the groups in the electro-acupuncture experiment

\begin{tabular}{llllllll}
$\mathbf{N}$ & $\begin{array}{l}\text { Gender } \\
(\mathbf{M} / \mathbf{F})\end{array}$ & Age $(\mathbf{y})$ & Height $(\mathbf{c m})$ & Weight $(\mathbf{k g})$ & BMI & $\begin{array}{l}\text { HR } \\
\text { the } \\
\text { experiment }\end{array}$ & Fear grade \\
\hline 52 & $26 / 26$ & $20.79 \pm 2.02$ & $166.92 \pm 8.10$ & $56.95 \pm 7.44$ & $20.37 \pm 1.52$ & $72.18 \pm 8.60$ & $4.94 \pm 1.55$ \\
\hline 53 & $25 / 28$ & $20.67 \pm 1.28$ & $165.55 \pm 8.51$ & $55.64 \pm 7.42$ & $20.13 \pm 1.26$ & $72.49 \pm 9.41$ & $5.00 \pm 1.56$ \\
& $0.084^{\#}$ & 0.385 & 0.848 & 0.903 & 0.856 & $0.603^{*}$ & $0.666^{*}$ \\
& 0.846 & 0.701 & 0.398 & 0.368 & 0.394 & 0.548 & 0.768 \\
\hline
\end{tabular}

${ }^{\#}$ Chi square value, ${ }^{*} \mathrm{Z}$ value, the rest are t test values; EA group, electro-acupuncture group.

\section{Comparison of HRV between the electro-acupuncture group and the control group}

During the electro-acupuncture, the SD, RMSSD and InHF of the electro-acupuncture group were all higher than those in the control group $(\mathrm{P}<0.017)$. The remaining indexes did not significantly differ regardless of whether they were from before the audio, after the audio or after the electro-acupuncture (Table 3; Fig. 5).

Table 3: Comparison of HRV between the electro-acupuncture group and the control group

\begin{tabular}{|c|c|c|c|c|c|}
\hline Indexes & Time & Control group & Electro-acupuncture group & $\mathbf{Z}$ & $\mathbf{P}$ \\
\hline \multirow{4}{*}{ HR } & 1 & $72.63 \pm 11.32$ & $71.32 \pm 10.99$ & -0.565 & 0.572 \\
\hline & 2 & $72.37 \pm 13.31$ & $70.68 \pm 12.25$ & -0.442 & 0.658 \\
\hline & 3 & $69.40 \pm 10.86$ & $66.189 \pm 11.37$ & -1.809 & 0.070 \\
\hline & 4 & $67.17 \pm 10.80$ & $65.98 \pm 10.75$ & -0.673 & 0.501 \\
\hline \multirow{4}{*}{ IBI } & 1 & $840.23 \pm 133.53$ & $854.83 \pm 131.98$ & -0.471 & 0.638 \\
\hline & 2 & $850.06 \pm 151.06$ & $868.75 \pm 148.86$ & -0.429 & 0.668 \\
\hline & 3 & $879.13 \pm 134.04$ & $924.23 \pm 147.72$ & -1.785 & 0.074 \\
\hline & 4 & $910.44 \pm 147.99$ & $927.09 \pm 146.78$ & -0.711 & 0.477 \\
\hline \multirow{4}{*}{ SD } & 1 & $64.12 \pm 33.98$ & $67.36 \pm 26.49$ & -1.064 & 0.287 \\
\hline & 2 & $51.23 \pm 20.75$ & $55.48 \pm 20.58$ & -1.279 & 0.201 \\
\hline & 3 & $54.90 \pm 19.97$ & $65.93 \pm 22.90$ & -2.500 & $0.012^{\star}$ \\
\hline & 4 & $60.40 \pm 28.91$ & $60.72 \pm 23.85$ & -0.333 & 0.739 \\
\hline \multirow{4}{*}{ RMSSD } & 1 & $57.38 \pm 40.23$ & $65.30 \pm 38.34$ & -1.307 & 0.191 \\
\hline & 2 & $47.34 \pm 34.38$ & $56.21 \pm 35.60$ & -0.942 & 0.346 \\
\hline & 3 & $46.35 \pm 20.76$ & $63.90 \pm 35.65$ & -2.544 & $0.011^{\star}$ \\
\hline & 4 & $51.86 \pm 25.33$ & $61.17 \pm 35.28$ & -0.920 & 0.358 \\
\hline \multirow{4}{*}{$\operatorname{lnVLF}$} & 1 & $7.02 \pm 1.15$ & $7.00 \pm 0.75$ & -0.122 & 0.903 \\
\hline & 2 & $6.34 \pm 0.78$ & $6.57 \pm 0.74$ & -1.320 & 0.187 \\
\hline & 3 & $6.76 \pm 0.84$ & $6.93 \pm 0.84$ & -1.179 & 0.238 \\
\hline & 4 & $7.08 \pm 1.15$ & $6.89 \pm 0.82$ & -1.199 & 0.231 \\
\hline \multirow{2}{*}{$\operatorname{lnLF}$} & 1 & $7.12 \pm 1.21$ & $7.31 \pm 0.82$ & -1.801 & 0.072 \\
\hline & 2 & $6.60 \pm 0.95$ & $6.81 \pm 0.85$ & -1.327 & 0.185 \\
\hline
\end{tabular}




\begin{tabular}{llllll}
\hline & 3 & $6.84 \pm 0.95$ & $7.06 \pm 0.77$ & -1.340 & 0.180 \\
& 4 & $6.92 \pm 1.06$ & $7.04 \pm 0.81$ & -0.538 & 0.590 \\
& 1 & $6.94 \pm 1.24$ & $7.20 \pm 1.03$ & -1.711 & 0.087 \\
$\ln \mathrm{HF}$ & 2 & $6.47 \pm 1.01$ & $6.87 \pm 0.98$ & -2.115 & $0.034^{\star}$ \\
& 3 & $6.67 \pm 0.81$ & $7.12 \pm 0.95$ & -2.615 & $0.009^{\star}$ \\
& 4 & $6.76 \pm 1.09$ & $6.98 \pm 1.01$ & -0.846 & 0.389 \\
& 1 & $1.43 \pm 0.82$ & $1.53 \pm 1.77$ & -1.201 & 0.230 \\
LF/HF & 2 & $1.55 \pm 1.39$ & $1.31 \pm 1.16$ & -0.831 & 0.406 \\
& 3 & $1.49 \pm 1.06$ & $1.35 \pm 1.37$ & -1.132 & 0.257 \\
& 4 & $1.71 \pm 1.54$ & $1.51 \pm 1.52$ & -0.850 & 0.395 \\
Triangular & 2 & $11.15 \pm 3.75$ & $14.15 \pm 4.14$ & -1.476 & 0.140 \\
Index & 3 & $12.38 \pm 3.96$ & $12.34 \pm 3.74$ & -1.591 & 0.112 \\
& 4 & $12.52 \pm 5.00$ & $13.85 \pm 4.40$ & -2.026 & $0.043^{\star}$ \\
& & $12.88 \pm 3.90$ & $12.84 \pm 4.40$ & -0.665 & 0.506 \\
\hline
\end{tabular}
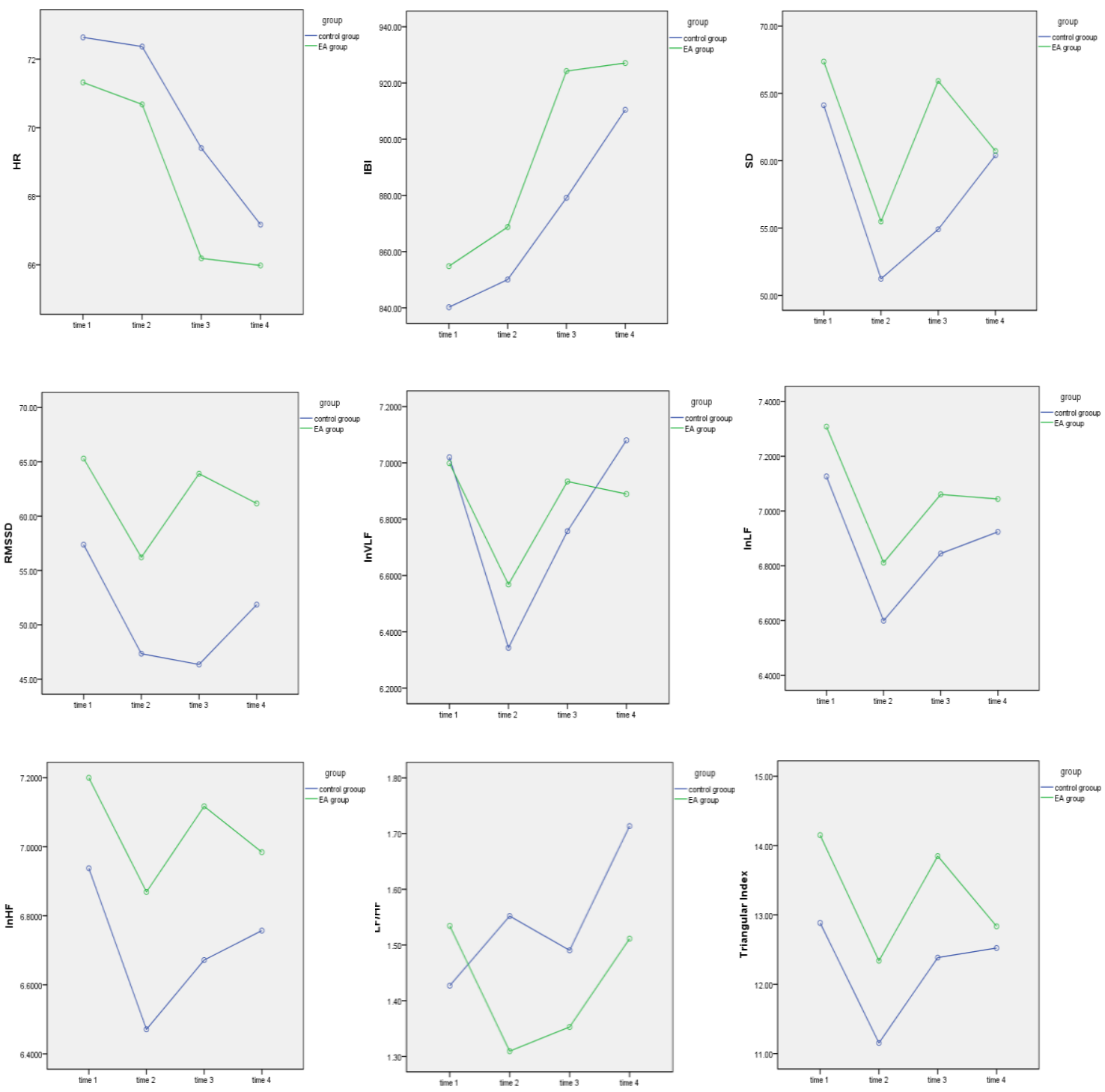

Figure 5: Changes in the 4 time points between the two groups

Time 1, before the audio, the 5-minute rest time before the audio. Time 2, after the audio, the last 5 minutes of the 20-minute audio. Time 3, during electro-acupuncture, the last 5 minutes of the 20-minute electro-acupuncture. Time 4, 
after electro-acupuncture, the last 5 minutes of the 20-minute quiet rest. * A Bonferroni-corrected Mann-Whitney test between the control group and the electro-acupuncture group, $\mathrm{p}<0.017$.

\section{Discussion}

Our result suggests that PC5 and PC6 electro-acupuncture may increase HRV in the short term, as indicated by the higher RMSSD and HF components. RMSSD is a time-domain index that reflects the activity of the cardiac parasympathetic system (Zahn et al., 2016). By contrast, the HF component is a frequency-domain index, mediated by the parasympathetic system (Anderson et al., 2012) and therefore is an indirect marker of the cardiac parasympathetic system (Billman, 2011). This may indicate that PC5 and PC6 electro-acupuncture was able to activate the cardiac parasympathetic nervous system.

It is established that the fear emotion can stimulate a fight-or-flight response, activate the sympathetic system, stimulate the secretion of adrenaline, and increase the HR, i.e., increase sympathetic and decreased parasympathetic activities (Aslaksen et al., 2007). In this study, the fear-invoking audio story stimulated the fight-or-flight response through the activated sympathetic system; subsequently, we electro-acupunctured at PC5 and PC6 on the scared subjects and found that a higher RMSSD and HF was produced, indicating that PC5 and PC6 electro-acupuncture could activate the parasympathetic system. The HR is controlled by the cardiac sympathetic and parasympathetic systems; activation of the parasympathetic system is supposed to reduce the HR, but in the present study, there was no significant reduction in HR as expected.

We suggest that this finding might be due to one or several of the following reasons: (1) The normal cardiac ANS always tries to balance the sympathetic and parasympathetic systems; when the parasympathetic system is activated, the sympathetic system will also activate to maintain the balance. (2) The effect of PC5 and PC6 electro-acupuncture was immediate; it has been proposed by Anderson et al. (2012) that acupuncture exerts its effect through physiological regulation including the improvement of HRV, synchronization of the cardiac sympathetic and parasympathetic systems and reduction in HR, which may occur secondary to sympathetic inhibition and/or parasympathetic activation. (3) There were large individual HRV differences among our healthy young subjects. (4) Factors affecting the HRV indexes (such as mental state, exercise, and sleep quality) that we did not control might have influenced the experiment. (5) The sample size of this study might have been too small.

In conclusion, PC5 and PC6 electro-acupuncture has an immediate effect on HRV in subjects who are exposed to fear-invoking auditory stimulation, increasing the RMSSD and HF component of HRV, which indicates that it may affect cardiac autonomic regulation, mainly via the parasympathetic system.

\section{Acknowledgements}

This was a cooperative project between the Southern Medical University and the University of Bergen. We thank all the volunteers for participating in this study.

\section{Author contributions}

Conceived and designed the experiments: Yong Huang, Gustav Wik, and Huanlin Huang. Performed the experiments: Huanlin Huang, Jizhou Wang, and Yujie Li. Analysed the data: Ole Bernt Fasmer and Zheng Zhong. 
Created the audio materials: Xinyi Zhang. Analysed the data and wrote the paper: Huanlin Huang and Shaohui Huang.

Conflict of Interest: All the authors have declared that they have no conflict of interest.

Funding: College students' innovative experimental project of Guangdong Province. No. 121211050

\section{References}

1. Anderson, B., Nielsen, A., Mckee, D., Jeffres, A. and Kligler, B. (2012) Acupuncture and heart rate variability: a systems level approach to understanding mechanism. Explore: The Journal of Science and Healing, 8, 99-106.

2. Asl, B. M., Setarehdan, S. K. and Mohebbi, M. (2008) Support vector machine-based arrhythmia classification using reduced features of heart rate variability signal. Artificial Intelligence in Medicine, 44, 51-64.

3. Aslaksen, P. M., Myrbakk, I. N., Høifødt, R. S. and Flaten, M. A. (2007). The effect of experimenter gender on autonomic and subjective responses to pain stimuli. Pain, 129, 260-268.

4. B A Cker, M., Grossman, P., Schneider, J., Michalsen, A., Knoblauch, N., Tan, L., Niggemeyer, C., Linde, K., Melchart, D. and Dobos, G. J. (2008) Acupuncture in migraine: investigation of autonomic effects. The Clinical journal of pain, 24, 106-115.

5. Billman, G. E. (2011) Heart rate variability - a historical perspective. Frontiers in Physiology, $2,86$.

6. Borkovec, T. D. and O'brien, G. T. (1977) Relation of autonomic perception and its manipulation to the maintenance and reduction of fear. Journal of Abnormal Psychology, 86, 163-171.

7. Bradley, M. M. and Lang, P. J. (1994) Measuring emotion: the Self-Assessment Manikin and the Semantic Differential. Journal of Behavior therapy and Experimental Psychiatry, 25, 49-59.

8. Brage, S., Brage, N., Franks, P. W., Ekelund, U. and Wareham, N. J. (2005) Reliability and validity of the combined heart rate and movement sensor Actiheart. European Journal of Clinical Nutrition, 59, 561-570.

9. de la Cruz Torres, B., Albornoz Cabello, M., García Bermejo, P. and Naranjo Orellana, J. (2016) Autonomic responses to ultrasound-guided percutaneous needle electrolysis of the patellar tendon in healthy male footballers. Acupuncture in Medicine, 34, 275-279.

10. Etzel, J. A., Johnsen, E. L., Dickerson, J., Tranel, D and Adolphs, R. (2006) Cardiovascular and respiratory responses during musical mood induction. International Journal of Psychophysiology, 61, 57-69.

11. Gullone, E., Cummins, R. A. and King, N. J. (1996) Self-reported fears: a comparison study of youths with and without an intellectual disability. Journal Of Intellectual Disability Research, 40 ( Pt 3), 227-240.

12. Guo, J. H. and Zhang, P. (2007) Ambulatory electrocardiography, People's Medical Publishing, Beijing. 12, 843-847.

13. Hsu, C. C., Weng, C. S., Sun, M. F., Shyu, L. Y., Hu, W. C. and Chang, Y. H. (2007) Evaluation of scalp and auricular acupuncture on EEG, HRV, and PRV. American Journal of Chinese Medicine, 35, 219-230.

14. Jia, B. A., Cheng, C. Y., Lin, Y. W., Li, T. C., Liu, H. J. and Hsieh, C. L. (2011) The $2 \mathrm{~Hz}$ and $15 \mathrm{~Hz}$ electroacupuncture induced reverse effect on autonomic function in healthy adult using a heart rate variability analysis. Journal of Traditional Complementary Medicine, 1, 51-56.

15. Li, M., Hu, L., Cai, R. L., Wu, Z. J. and Wang, K. M. (2012) [Effects of electroacupuncture at PC6 and BL15 on nerve electrical activity in spinal dorsal root and norepinephrine and dopamine contents in paraventricular nucleus of hypothalamus in rats with acute myocardial ischemia]. Zhong Xi Yi Jie He Xue Bao, 10, 874-879.

16. Litscher, G. (2010) Transcontinental and translational high-tech acupuncture research using computer-based heart rate 
and "Fire of Life" heart rate variability analysis. Journal of acupuncture and meridian studies, 3, 156-164.

17. Liu, Q., Yang, L., Li, Z. H., Lu, G. and Sun, J. H. (2002) Study on homologus relationship of neuro-projection between heart and Neiguan point area with non-fluorescence double labeling technique in rat. Chinese Journal of Anatomy, $39-42$.

18. Liu, T. S., Luo, Y. J., Ma, H. and Huang, Y. X. (2006) The Establ ishment and Assessment of a Native Affective Sound System. PSYCHOLOGICAL SCIENCE, 406-408.

19. Momen, A., Bower, D., Leuenberger, U. A., Boehmer, J., Lerner, S., Alfrey, E. J., Handly, B. and Sinoway, L. I. (2005) Renal vascular response to static handgrip exercise: sympathetic vs. autoregulatory control. American Journal of Physiology Heart Circulatory Physiology, 289, H1770-H1776.

20. Nakahara, H., Kawada, T., Ueda, S. Y., Kawai, E., Yamamoto, H., Sugimachi, M. and Miyamoto, T. (2016) Electroacupuncture most effectively elicits depressor and bradycardic responses at $1 \mathrm{~Hz}$ in humans. Clinical Autonomic Research, 26, 59-66.

21. Palomba, D., Sarlo, M., Angrilli, A., Mini, A. and Stegagno, L. (2000) Cardiac responses associated with affective processing of unpleasant film stimuli. International Journal of Psychophysiology, 36, 45-57.

22. Park, S., Jung, W., Moon, S., Park, J. M., Ko, C., Cho, K., Kim, Y. and Bae, H. (2008) Effects of acupuncture on autonomic nervous system in normal subjects under mental stress. Journal of Korean Oriental Medical Ophthalmology and Otolaryngology and Dermatology, 29, 107-115.

23. Tao, Z. L., Li, D. W., Zhang, J. J. and Li, Q. (1993) The Segmental Distribution of Sympathetic Afferent Neurons of the Heart, Cardiac Nerve and Projection of the Cardiac Nerve to the Central Nerous System. Acupuncture Research, 257-261.

24. Wang, J. D., Kuo, T. B. and Yang, C. C. (2002) An alternative method to enhance vagal activities and suppress sympathetic activities in humans. Autonomic Neuroscience, 100, 90-95.

25. Witt, C. M., Meissner, K., Pach, D., Thiele, C., Ludtke, R., Ghadiyali, Z., Deter, H. C. and Zimmermann-Viehoff, F. (2012) Stimulation of gastric slow waves with manual acupuncture at acupuncture points ST36 and PC6--a randomized single blind controlled trial. Journal of Neurogastroenterology and Motility, 24, 438-445, e211-e212.

26. World Health Organization (2008) WHO Standard Acupuncture Point Locations in the Western Pacific Region. World Health Organization: Regional Office for the Western Pacific.

27. Xue, Y. Y., Wang, J. T., Yang, B., Zhang, Y. and Zhang, Y. (2009) Study of some physiological indexes and cardiac autonomic outflow patterns of fear emotion elicited by audio material. Chinese Journal Behavioral Medicine and Brain Science, 2, 145-147.

28. Zahn, D., Adams, J., Krohn, J., Wenzel, M., Mann, C. G., Gomille, L. K., Jacobi-Scherbening, V. and Kubiak, T. (2016) Heart rate variability and self-control-A meta-analysis. Biological Psychology, 115, 9-26. 